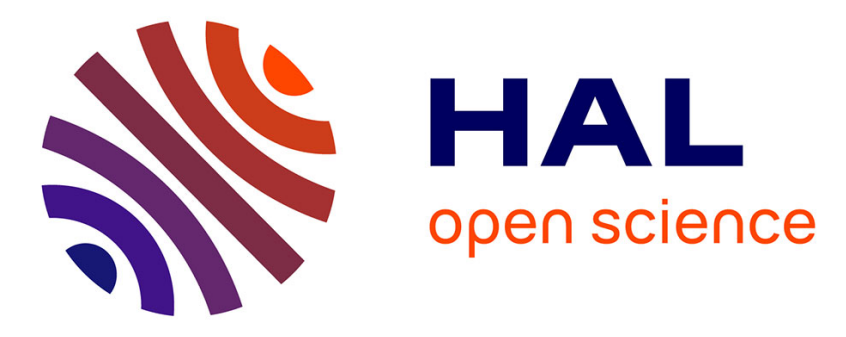

\title{
First-principles molecular-dynamics simulations for neutral p-chloranil and its radical anion
}

Claudine Katan, P. E. Blöchl, P. Margl, Christiane Koenig

\section{To cite this version:}

Claudine Katan, P. E. Blöchl, P. Margl, Christiane Koenig. First-principles molecular-dynamics simulations for neutral p-chloranil and its radical anion. Physical Review B: Condensed Matter and Materials Physics (1998-2015), 1996, 53 (18), pp.12112-12120. 10.1103/PhysRevB.53.12112 . hal00880748

\section{HAL Id: hal-00880748 \\ https://hal.science/hal-00880748}

Submitted on 6 Nov 2013

HAL is a multi-disciplinary open access archive for the deposit and dissemination of scientific research documents, whether they are published or not. The documents may come from teaching and research institutions in France or abroad, or from public or private research centers.
L'archive ouverte pluridisciplinaire $\mathbf{H A L}$, est destinée au dépôt et à la diffusion de documents scientifiques de niveau recherche, publiés ou non, émanant des établissements d'enseignement et de recherche français ou étrangers, des laboratoires publics ou privés. 


\title{
First-principles molecular-dynamics simulations for neutral $p$-chloranil and its radical anion
}

\author{
C. Katan \\ Groupe Matière Condensée et Matériaux, Université Rennes I, Campus de Beaulieu, F-35042 Rennes Cedex, France \\ P. E. Blöchl \\ IBM Research Division, Zurich Research Laboratory, CH-8803 Rüschlikon, Switzerland \\ P. Margl \\ Department of Chemistry, University of Calgary, 2500 University Drive N.W., Calgary, Alberta, Canada T2N 1N4 \\ C. Koenig \\ Groupe Matière Condensée et Matériaux, Université Rennes I, Campus de Beaulieu, F-35042 Rennes Cedex, France
}

(Received 10 July 1995)

\begin{abstract}
The neutral $p$-chloranil (2,3,5,6-tetrachloro- $p$-benzoquinone) and its radical anion have been extensively studied using the Car-Parrinello projector augmented wave method, which is an all-electron electronic structure method for first-principles molecular dynamics based on the local density approximation of density functional theory. Frequencies and eigenmodes are derived by fitting a system of harmonic oscillators to the moleculardynamics trajectories. The dependence of the bond lengths and vibrational frequencies on the molecular ionicity is discussed, and the electron affinity, Coulomb repulsion, and the spin-splitting parameter of $p$-chloranil are also derived. [S0163-1829(96)05318-0]
\end{abstract}

\section{INTRODUCTION}

Organic $\pi$-molecular donors and acceptors often form charge transfer (CT) complexes consisting of either separate donor and acceptor stacks or mixed stacks in which donors and acceptors alternate along the stacking axis. Although a majority of the CT complexes crystallize in this latter insulating mixed-stack structure, research interest has mainly been focused on the segregated compounds as they can exhibit a high electrical conductivity. ${ }^{1-3}$ But more recently, the discovery of the so-called neutral-to-ionic phase transition has renewed interest in the mixed-stack compounds. ${ }^{4}$

For a long time, the underlying assumption has been that mixed-stack complexes can only be either completely neutral or completely ionic. ${ }^{5}$ However, extensive experimental studies of these systems in the early eighties led to the development of a theoretical model that allows a degree of ionicity different from zero or one. ${ }^{6,7}$ Furthermore, one of the essential requirements so that the segregated CT complexes yield high conductivity is that the charge transfer from donors to acceptors be incomplete. Therefore, attention has focused on obtaining an estimate for this quantity. Among the various methods proposed there are two based on the dependence of the bond lengths on the molecular ionicity ${ }^{8}$ and intramolecular vibrational frequencies. ${ }^{9-12}$ Until now, the correlations between these parameters were deduced from experimental data obtained with a set of compounds expected to have known oxidation states.

Owing to its electron affinity, $p$-chloranil (2,3,5,6tetrachloro- $p$-benzoquinone, CA) belongs to the family of molecules that forms CT complexes with donors such as TTF (tetrathiafulvalene), DMTTF or TMPD. ${ }^{13}$ Most of these compounds have a mixed-stack crystalline structure, and some of them exhibit a neutral-to-ionic phase transition. The evolution of the degree of charge transfer versus temperature or pressure, which is crucial information for the experimental analysis and modeling of such transitions, has partly been deduced from an assumed linear ${ }^{11}$ or nearly linear ${ }^{12}$ behavior of some intramolecular vibration frequencies of CA versus ionicity. However, for CA this assumption cannot be verified experimentally owing to the lack of synthesized compounds in which a partial degree of ionicity is fixed by stoichiometry. With the development of powerful molecular-dynamics (MD) methods it has recently become possible to investigate this fundamental point theoretically using numerical simulations.

In this work we have undertaken a detailed analysis of the static and dynamic properties of $\mathrm{CA}$ in various oxidation states with the Car-Parrinello projector augmented wave (CP-PAW) method. ${ }^{14}$ This is the verification of the linearity of the frequency and bond length variation versus the molecular ionicity of CA.

The CP-PAW method combines the first-principles MD method of Car and Parrinello ${ }^{15,16}$ with an all-electron electronic structure method that can be efficiently applied to all elements of the Periodic Table within the local density approximation (LDA) of density functional theory. Vibrational analysis is performed by fitting a system of harmonic oscillators to the MD trajectories, ${ }^{17}$ a method that has recently been used to investigate the static and dynamic properties of ferrocene.

This paper is organized as follows: in Sec. II we summarize the basic concepts of both the CP-PAW method and the method used for vibrational analysis. Section III contains the results of the calculations for neutral CA: the ground state, electronic structure properties, and vibrations. In Sec. IV we analyze the influence the ionicity of the molecule has on interatomic distances and vibrational frequencies, and derive 


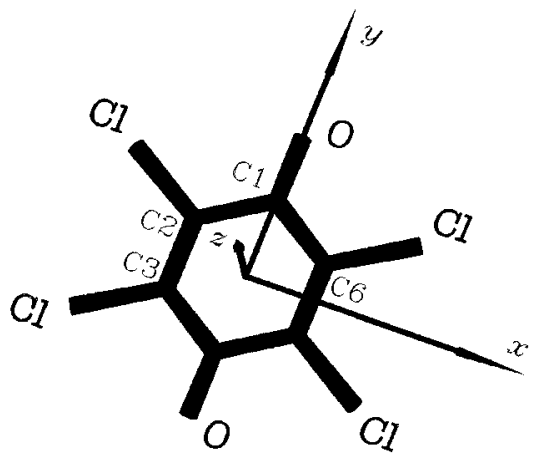

FIG. 1. Chloranil molecule.

an estimate of the electron affinity, the Coulomb repulsion, and the spin-splitting parameter of $\mathrm{CA}$.

\section{THEORETICAL BACKGROUND}

\section{A. Car-Parrinello projector augmented wave method}

Electronic structure and atomic trajectories calculations of CA have been performed with the CP-PAW method. A detailed description of this scheme developed by one of us can be found elsewhere. ${ }^{14}$ This method combines the original fictitious Lagrangian approach of Car and Parrinello, ${ }^{15,16}$ in which the atomic coordinates and the electronic wave function are treated simultaneously by a set of Newton's equations, with an all-electron (AE) electronic structure scheme that combines the versatility and accuracy of the linearized augmented plane wave (LAPW) method $^{18}$ with the formal simplicity of the traditional plane-wave pseudopotential approach. ${ }^{19}$ It allows high-quality, energy-conserving, firstprinciples MD simulations even for large systems.

The core wave functions are imported from isolated atoms according to the frozen-core approximation. The valence wave function is decomposed in the following way:

$$
|\psi\rangle=|\widetilde{\psi}\rangle+\left(\left|\psi^{1}\right\rangle-\left|\widetilde{\psi}^{1}\right\rangle\right)
$$

where $|\widetilde{\psi}\rangle$ is a smooth so-called pseudo (PS)-wave function, which is equal to the AE wave function except in the atomic regions $\Omega_{R}$. This slowly-varying PS wave function is expanded into plane waves. Within the core region, $|\widetilde{\psi}\rangle$ is a smooth continuation from the outside. Hence, $\left|\psi^{1}\right\rangle-\left|\widetilde{\psi}^{1}\right\rangle$ is a remainder that contains all strong oscillations of the atomic node structure. It is localized in $\Omega_{R}$ and can thus be represented as sums of one-center partial wave expansions:

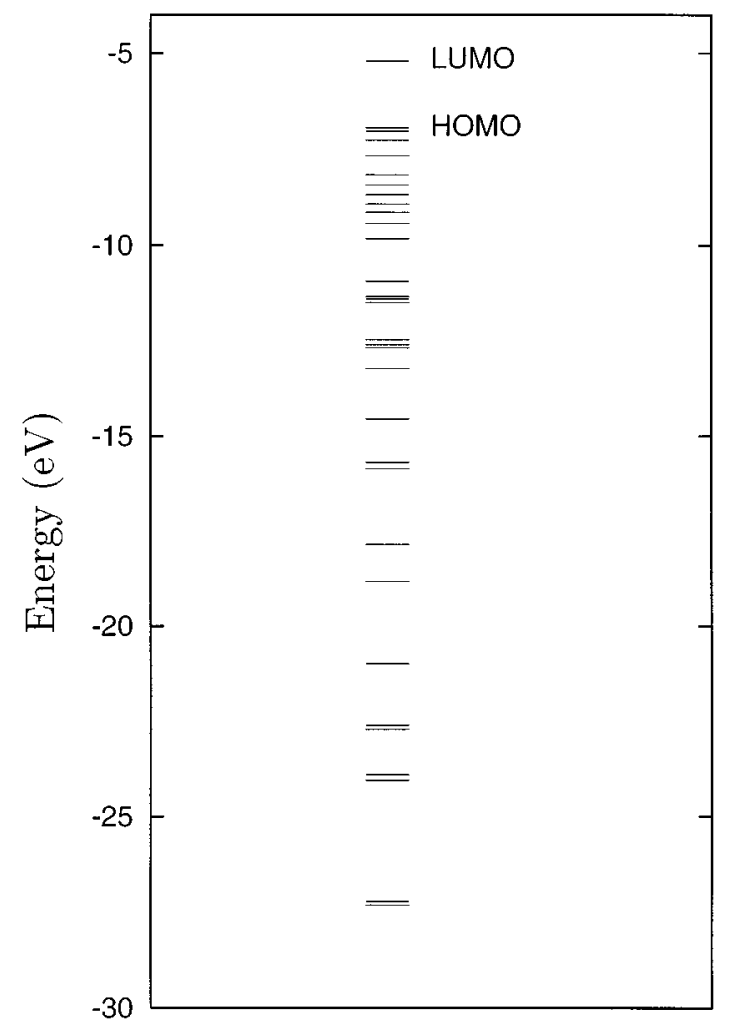

FIG. 2. Molecular energy levels of chloranil.

$$
\begin{aligned}
& \left|\psi^{1}\right\rangle=\sum_{i}\left|\phi_{i}\right\rangle c_{i}, \\
& \left|\widetilde{\psi}^{1}\right\rangle=\sum_{i}\left|\widetilde{\phi}_{i}\right\rangle c_{i} .
\end{aligned}
$$

Here $\left|\widetilde{\psi}^{1}\right\rangle=|\widetilde{\psi}\rangle$ in the augmentation region and $\left|\widetilde{\psi}^{1}\right\rangle=\left|\psi^{1}\right\rangle$ outside it, when assuming no truncation of the expansions. The function $\left|\psi^{1}\right\rangle$ corresponds to one-center expansions of the $\mathrm{AE}$ valence wave function. A natural choice for the $\mathrm{AE}$ partial waves $\left|\phi_{i}\right\rangle$ is to take them as the solutions of the radial Schrödinger equation for the isolated atoms that are orthogonalized to the core states. Hence the index $i$ refers to the atomic site $R$, the angular momentum quantum numbers $L=(l, m)$ and an additional index $n$ which distinguishes different partial waves having the same site and angular momentum indices. The corresponding PS partial waves $\left|\widetilde{\phi}_{i}\right\rangle$ are slowly-varying functions identical to $\left|\phi_{i}\right\rangle$ outside $\Omega_{R}$. The coefficients $c_{i}$ are obtained as scalar product of the PS wave function and a suitable atom-centered projector function.

This decomposition defines a linear transformation between the physical AE wave function and the PS wave func-

TABLE I. Calculated bond lengths (in $\AA$ ) and angles (in degrees) for the chloranil molecule in comparison with experiments on the crystal structure of chloranil.

\begin{tabular}{lccccccccc}
\hline \hline & $\mathrm{C}_{1}-\mathrm{O}$ & $\mathrm{C}_{1}-\mathrm{C}_{2}$ & $\mathrm{C}_{2}-\mathrm{C}_{3}$ & $\mathrm{C}_{2}-\mathrm{Cl}$ & $\mathrm{C}_{2} \mathrm{C}_{1} \mathrm{C}_{6}$ & $\mathrm{C}_{2} \mathrm{C}_{1} \mathrm{O}$ & $\mathrm{C}_{1} \mathrm{C}_{2} \mathrm{C}_{3}$ & $\mathrm{C}_{1} \mathrm{C}_{2} \mathrm{Cl}$ & $\mathrm{ClC}_{2} \mathrm{C}_{3}$ \\
\hline CP-PAW & 1.219 & 1.477 & 1.350 & 1.671 & 117.9 & 121.1 & 121.1 & 116.3 & 122.6 \\
Ref. 31 & 1.211 & 1.490 & 1.344 & 1.701 & 117.4 & 121.3 & 121.2 & 115.9 & 122.9 \\
Ref. 32 & 1.195 & 1.477 & 1.342 & 1.714 & 117.3 & 121.4 & 121.4 & 116.2 & 122.4 \\
\hline \hline
\end{tabular}



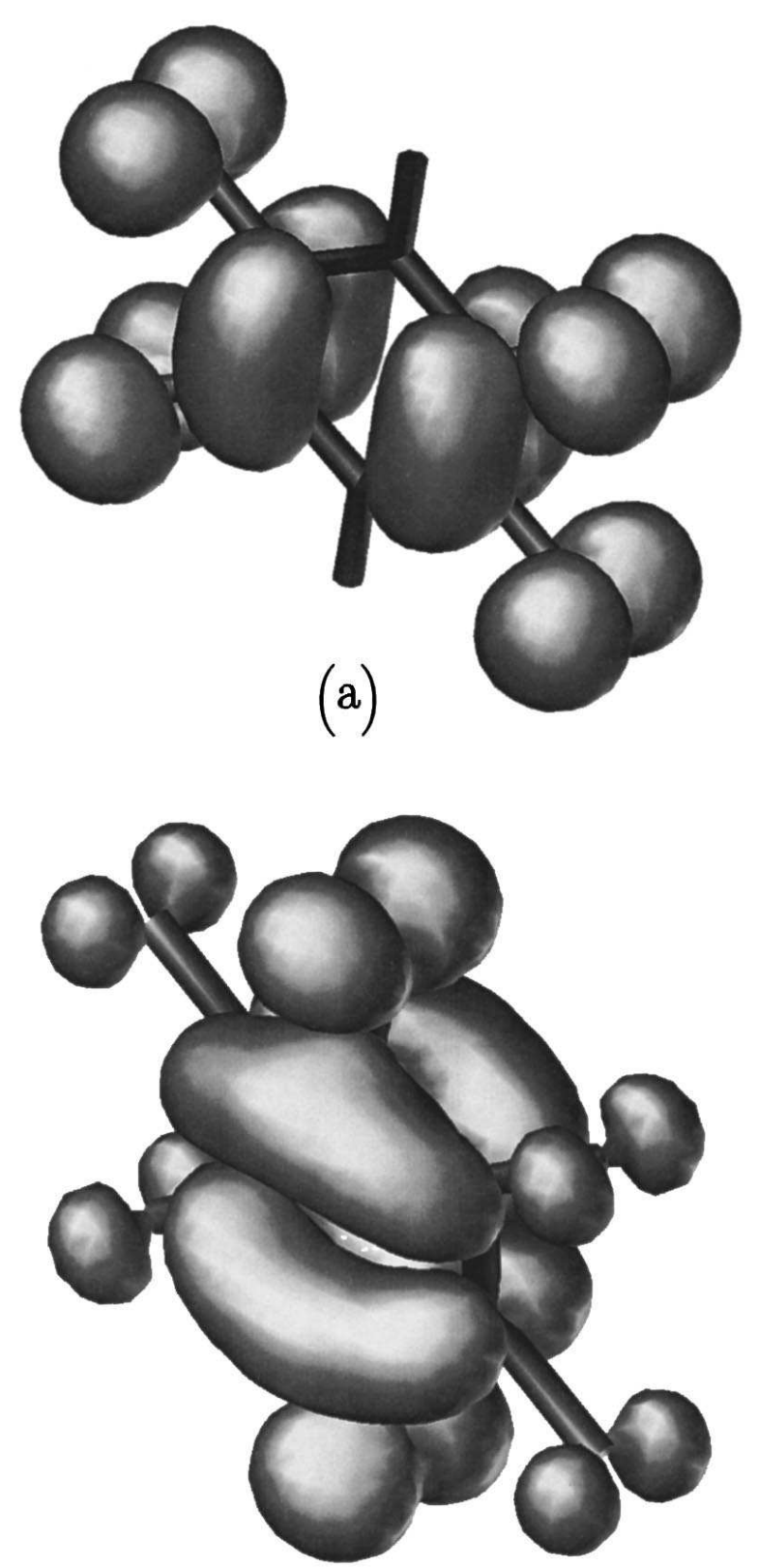

(b)

FIG. 3. Isodensity representation of (a) the HOMO and (b) the LUMO of the chloranil molecule.

tion so that expectation values such as the total energy or charge densities can be directly evaluated as a functional of the PS wave function. This results in numerical efficiency for the calculation of the Hamiltonian and forces, and firstprinciples MD can then be achieved in a straightforward manner.

\section{B. Vibrational analysis}

The atomic trajectories obtained by MD simulations are analyzed in terms of harmonic vibrations using the technique developed by Margl and co-workers. ${ }^{17,20}$ This approach, which is similar to Kohanoff's ${ }^{21}$ with the exception of the penalty function used for the least-squares fit, fits the trajectories of a harmonic system to those obtained from the MD simulation. The fit parameters are the eigenvectors of the dynamical matrix, the amplitudes, and the frequencies. Here we only summarize the basic steps of this scheme; a more detailed description can be found elsewhere. ${ }^{17,20}$

First, one has to remove the angular momentum of the entire molecule from the original trajectory. Then the resulting trajectory is split into mutually orthogonal subtrajectories for each given irreducible representation of the point group of the molecule under consideration, by applying the character projection operators. In order to start the fitting procedure for a specific subtrajectory, a set of initial frequencies is estimated from the Fourier transform of the velocity autocorrelation function. Eigenvectors and amplitudes are estimated from the sine and cosine Fourier transforms of the subtrajectory. Then, the least-squares fit is achieved by minimizing the penalty functional

$$
Q=\frac{1}{T} \int_{0}^{T} d t\left[\sum_{R} m_{R}\left[\mathbf{v}_{\mathbf{R}}(t)-\widetilde{\mathbf{v}}_{\mathbf{R}}(t)\right] \cdot\left[\mathbf{v}_{\mathbf{R}}(t)-\widetilde{\mathbf{v}}_{\mathbf{R}}(t)\right]\right]
$$

under the constraint of orthogonal eigenvectors. Here $T$ is the simulation time and $m_{R}$ the mass of the atom at site $R$. The vectors $\mathbf{v}_{\mathbf{R}}(t)$ and $\widetilde{\mathbf{v}}_{\mathbf{R}}(t)$ refer to the velocities of this atom in the simulation and in the trial system, respectively. A minimum of $Q$ is obtained in an iterative procedure and leads to a self-consistent set of eigenvectors, amplitudes, and frequencies that optimally fits the subtrajectory.

\section{Computational details}

The pseudopotential, partial waves, and projector functions have been constructed with the procedure described in Ref. 14 for nontransition metals, using $r_{k}=1.0$ for oxygen and carbon, $r_{k}=1.2$ for chlorine, and $\lambda=6\left(r_{k}\right.$ and $\lambda$ are defined in Ref. 14). One projector function per angularmomentum quantum number was used for $s$ - and $p$-angular momenta. The PS wave functions were expanded into plane waves up to a kinetic energy cutoff of $30 \mathrm{Ry}$. Tests were made to ensure that this was sufficient even for oxygen. Most of the calculations were non-spin-polarized and within the LDA, using the LDA parametrization by Perdew and Zunger $^{22}$ based on Monte Carlo simulations of the free electron gas investigated by Ceperley and Adler. ${ }^{23}$ Nevertheless, a few spin-polarized calculations were done to calculate the spin-splitting parameter and to check the validity of the estimates obtained for the electron affinity. We also performed calculations that included two types of gradient correction: one with a Becke gradient correction to the exchange energy alone $^{24}$ (denoted GC1), the other with an additional Perdew gradient correction for the correlation energy ${ }^{25}$ (denoted GC2). The molecule was oriented with the ring perpendicular to the $z$ axis and the $\mathrm{C}=\mathrm{O}$ bonds parallel to the $y$ axis (Fig. 1). The calculations were carried out using a tetragonal supercell of $20 \times 20 \times 15$ a.u. ${ }^{3}$ containing one molecule. In all calculations the molecule had been electrostatically decoupled from its periodic images as described in Ref. 26, and by increasing the lattice vectors it was verified that this supercell was sufficiently large to allow a good description of an isolated molecule. 


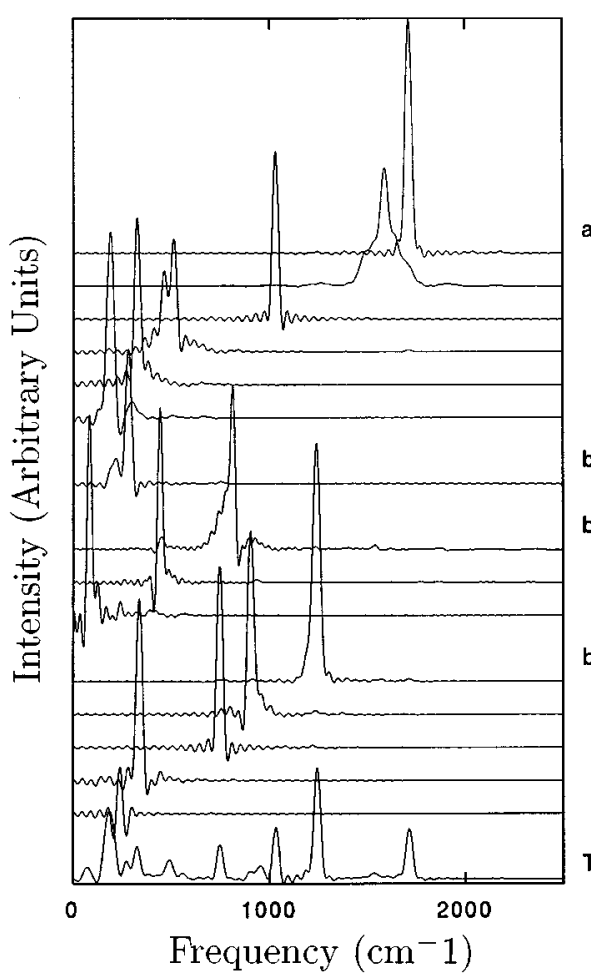

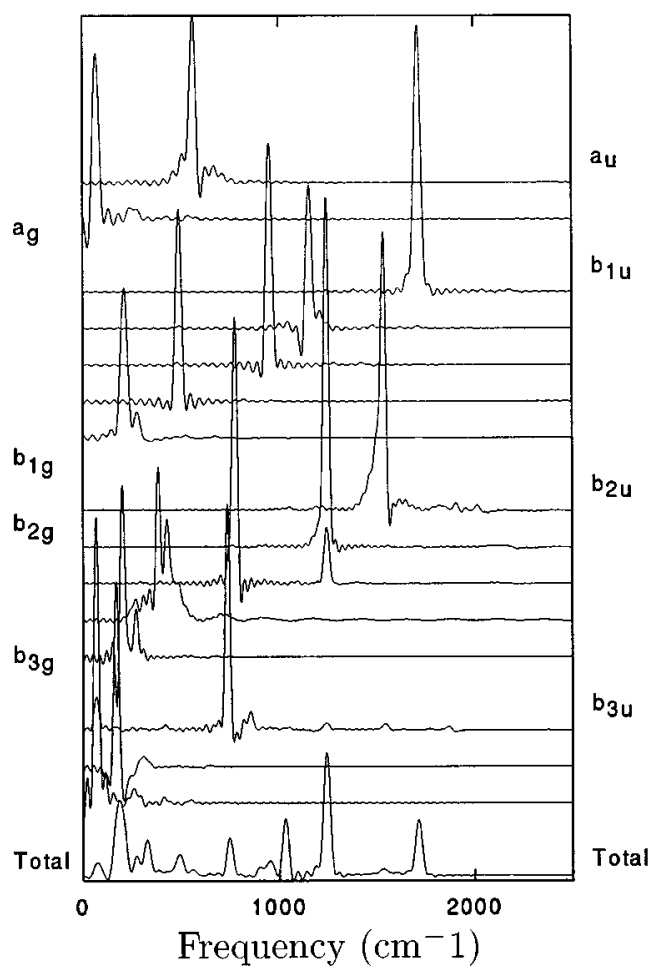

FIG. 4. Plots of projection of the subtrajectories onto each eigenmode of the corresponding irreducible representation. Bottom: total vibrational spectrum of chloranil.
The initial geometry for the MD simulations was obtained from the optimized geometry of the molecule in its ground state by adding a random distribution of the atomic velocities and letting the system equilibrate at $30 \mathrm{~K}$. During the MD simulation, an average temperature of $30 \mathrm{~K}$ was maintained using a Nosé thermostat ${ }^{27-29}$ with an eigenfrequency of 15 THz. The Verlet algorithm ${ }^{30}$ was used with a time step of 10 a.u. and a total simulation time of 1.1 or $1.5 \mathrm{ps}$. To prevent deviations from the Born-Oppenheimer surface, we also applied a small constant friction to the dynamics of the electronic wave functions.

For the vibrational analysis we used the molecular point group $D_{2 h}$, to which CA belongs. The accuracy of the vibrational modes was checked by examining the vibrational spectrum obtained when projecting the subtrajectory onto a specified eigenvector. For a satisfactory set of eigenvectors, each projection should yield a single, well-defined peak. Moreover, we verified that the residual trajectory remaining after all modes had been removed was comprised only of noise.

\section{NEUTRAL CHLORANIL}

\section{A. Ground-state properties of chloranil}

To our knowledge, this is the first $a b$ initio study of the ground-state and vibrational properties of CA. The optimized geometry at a temperature of $0 \mathrm{~K}$ is given in Table I; it was found to belong to the $D_{2 h}$ molecular point group. For this chloroderivative of $p$-benzoquinone, we observed no tendency to be nonplanar. The calculated bond lengths and angles of the molecule compare well with the values found in the crystal structure of CA at $110 \mathrm{~K}$ (Ref. 31) and the less precise room-temperature values. ${ }^{32}$ Nevertheless, it should be noted that within the LDA, the calculated $\mathrm{C}-\mathrm{Cl}$ bond length seems to be somewhat small (by $2 \%$ ) compared to the experimental results. When including a gradient correction (GC1 or GC2), this distance was found to be about $3 \%$ larger, i.e., much closer to the experimental values, whereas the differences for the other distances were less than $1 \%$.

The molecular energy levels of CA are plotted in Fig. 2. Both the highest occupied molecular orbital (HOMO) and

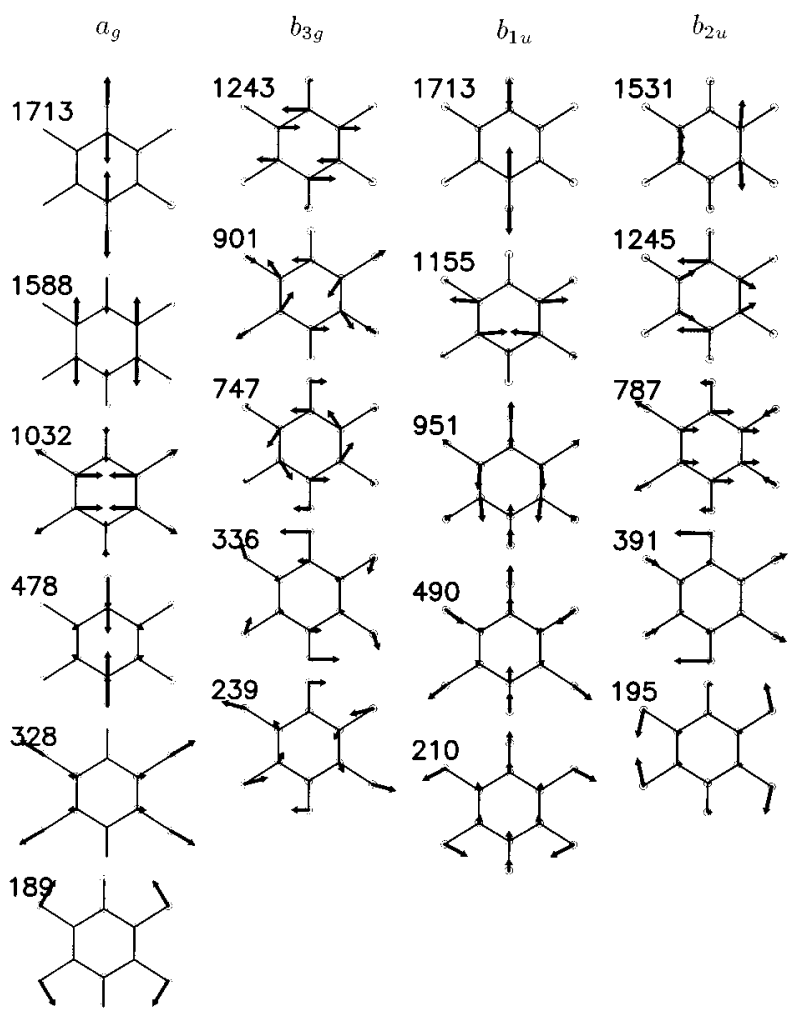

FIG. 5. Calculated in-plane eigenvectors of chloranil. The numbers refer to the corresponding frequencies in $\mathrm{cm}^{-1}$. 
the lowest unoccupied molecular orbital (LUMO) states are built primarily from atomic $p$ orbitals perpendicular to the molecular plane. The HOMO is essentially localized on the chlorine atoms and the neighboring carbon atoms, whereas the LUMO has a significant weight on the $\mathrm{C}-\mathrm{O}$ bonds, as can be seen in Figs. 3(a) and 3(b), respectively. The fact that the LUMO state has a large weight on the $\mathrm{C}-\mathrm{O}$ (anti-)bonds explains why these bond lengths and associated vibrational frequencies exhibit the strongest dependence on the ionicity of the molecule. This point will be discussed further in the next section.

\section{B. Vibrations}

We determined the modes and vibrational frequencies of CA from two MD simulations at $30 \mathrm{~K}$ for 1.1 and $1.5 \mathrm{ps}$ using the technique as briefly described in Sec. II B. The symmetry of the vibrational modes of CA was treated in the context of the $D_{2 h}$ molecular point group. The thirty fundamental vibrations of $\mathrm{CA}$ are classified into

$$
6 a_{g}+1 b_{1 g}+3 b_{2 g}+5 b_{3 g}+2 a_{u}+5 b_{1 u}+5 b_{2 u}+3 b_{3 u},
$$

using the convention commonly found in the literature. ${ }^{33}$ The vibrations belonging to $b_{1 g}, b_{2 g}, a_{u}$, and $b_{3 u}$ representations are out of plane, whereas those belonging to $a_{g}, b_{3 g}, b_{1 u}$, and $b_{2 u}$ are in-plane modes.

After removing the angular momentum of the entire molecule, we split the complete trajectory into eight mutually orthogonal partial trajectories belonging to the different irre-

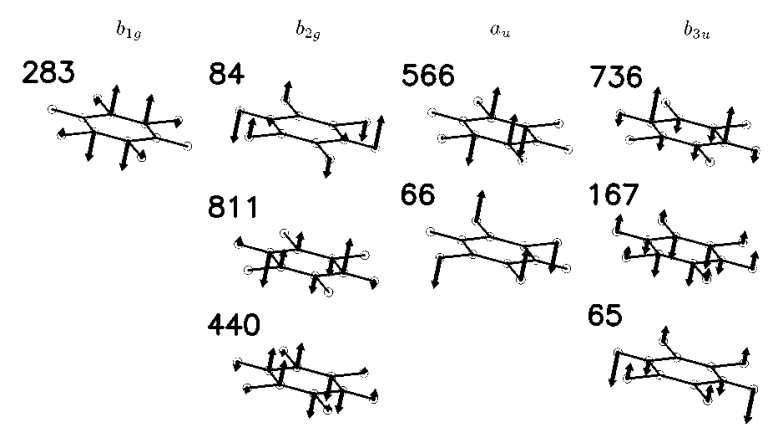

FIG. 6. Calculated out-of-plane eigenvectors of chloranil. The numbers refer to the corresponding frequencies in $\mathrm{cm}^{-1}$.

ducible representations. For each subtrajectory, the fitting procedure was used to determine the eigenmodes and eigenfrequencies of that symmetry. The consistency of the decomposition was checked by projecting the subtrajectory onto each eigenmode of the corresponding irreducible representation. The resulting spectra are plotted in Fig. 4. They clearly show that almost all modes have been well separated. The eigenvectors corresponding to thirty independent vibrational modes are plotted in Figs. 5 (in-plane vibrations) and 6 (outof-plane vibrations). Their eigenfrequencies are given in Tables II and III.

Most of the experimental results of vibrational frequencies in CA were obtained in the crystalline state. ${ }^{33-37}$ These frequencies may differ from those calculated here owing to the influence of the intermolecular interactions. Neverthe-

TABLE II. Calculated in-plane vibrational frequencies $\left(\right.$ in $\left.\mathrm{cm}^{-1}\right)$ of chloranil in comparison with experiment. All experiments used crystalline chloranil unless otherwise indicated. $\Delta$ represents the deviation of the calculated values from the experimental results in Ref. 34 .

\begin{tabular}{|c|c|c|c|c|c|c|c|}
\hline \multicolumn{2}{|c|}{ Symm. species } & \multirow{2}{*}{$\frac{\text { CP-PAW }}{1713}$} & \multirow{2}{*}{$\begin{array}{c}\text { Ref. } 34 \\
1693\end{array}$} & \multirow{2}{*}{$\begin{array}{c}\text { Ref. } 33 \\
1690\end{array}$} & \multirow{2}{*}{$\frac{\text { Ref. } 35}{1653^{b}}$} & \multirow[t]{2}{*}{ Ref. 36} & \multirow{2}{*}{$\frac{\Delta \%}{2}$} \\
\hline$a_{g}$ & $\nu_{1}$ & & & & & & \\
\hline & $\nu_{2}$ & 1588 & 1609 & 1610 & & & 2 \\
\hline & $\nu_{3}$ & 1032 & 1007 & 1004 & & & 3 \\
\hline & $\nu_{4}$ & 478 & 496 & 491 & & & 4 \\
\hline & $\nu_{5}$ & 328 & 330 & 325 & & & 1 \\
\hline & $\nu_{6}$ & 189 & 200 & 196 & & & 6 \\
\hline \multirow[t]{5}{*}{$b_{3 g}$} & $\nu_{11}$ & 1243 & 1247 & 1270 & $1211,{ }^{b} 1218^{c}$ & & 1 \\
\hline & $\nu_{12}$ & 901 & 852 & 1247 & & & 6 \\
\hline & $\nu_{13}$ & 747 & (779) $759^{a}$ & 852 & & & (5) 2 \\
\hline & $\nu_{14}$ & 336 & 307 & 302 & & & 9 \\
\hline & $\nu_{15}$ & 239 & 267 & 262 & & & 12 \\
\hline \multirow[t]{5}{*}{$b_{1 u}$} & $\nu_{18}$ & 1713 & 1685 & 1688 & $1695,{ }^{b} 1703^{c}$ & $1710^{\mathrm{c}}$ & 2 \\
\hline & $\nu_{19}$ & 1155 & 1110 & 1111 & $1115,{ }^{b} 1146^{c}$ & $1115^{\mathrm{c}}$ & 4 \\
\hline & $\nu_{20}$ & 951 & 908 & 905 or 1111 & & $896^{\mathrm{c}}$ & 5 \\
\hline & $\nu_{21}$ & 490 & 473 & 714 & $742^{\mathrm{c}}$ & & 4 \\
\hline & $\nu_{22}$ & 210 & (186) $184^{\mathrm{a}}$ & 194 & & & 12 \\
\hline \multirow[t]{5}{*}{$b_{2 u}$} & $\nu_{23}$ & 1531 & 1572 & 1572 & $1560^{\mathrm{b}, \mathrm{c}}$ & $1548^{\mathrm{c}}$ & 3 \\
\hline & $\nu_{24}$ & 1245 & 1260 & 1260 & $1243^{c}$ & $1239^{c}$ & 2 \\
\hline & $\nu_{25}$ & 787 & 755 & 755 & $1115^{\mathrm{b}}$ & & 4 \\
\hline & $\nu_{26}$ & 391 & 376 & 373 & & & 4 \\
\hline & $\nu_{27}$ & 195 & 206 & 176 & & & 6 \\
\hline
\end{tabular}

${ }^{\mathrm{a} C a l c u l a t e d}$ frequencies.

${ }^{\mathrm{b}}$ Solution phase.

${ }^{\mathrm{c}}$ Vapor phase. 
TABLE III. Calculated out-of-plane vibrational frequencies (in $\mathrm{cm}^{-1}$ ) of chloranil in comparison with experiments on the chloranil crystal. All experiments used crystalline chloranil unless otherwise indicated. $\Delta$ represents the deviation of the calculated values from the experimental results in Ref. 34.

\begin{tabular}{|c|c|c|c|c|c|c|c|}
\hline $\begin{array}{l}\text { Symm. } \\
\text { species }\end{array}$ & & CP-PAW & Ref. 34 & Ref. 33 & Ref. 35 & Ref. 36 & $\Delta \%$ \\
\hline$b_{1 g}$ & $\nu_{7}$ & 283 & 341 & 222 & & & 20 \\
\hline \multirow[t]{3}{*}{$b_{2 g}$} & $\nu_{8}$ & 811 & 779 & 776 & & & 4 \\
\hline & $\nu_{9}$ & 440 & 422 & 420 & & & 4 \\
\hline & $\nu_{10}$ & 84 & 141 & 136 & & & 68 \\
\hline \multirow[t]{2}{*}{$a_{u}$} & $\nu_{16}$ & 566 & $431^{\mathrm{a}}$ & & & & 24 \\
\hline & $\nu_{17}$ & 66 & $56^{\mathrm{a}}$ & & & & 15 \\
\hline \multirow[t]{3}{*}{$b_{3 u}$} & $\nu_{28}$ & 736 & 714 & 470 & $453^{\mathrm{b}}$ & $737^{\mathrm{c}}$ & 3 \\
\hline & $\nu_{29}$ & 167 & 186 & 125 & & & 12 \\
\hline & $\nu_{30}$ & 65 & 88 & 80 & & & 35 \\
\hline
\end{tabular}

${ }^{\mathrm{a} C a l c u l a t e d}$ frequencies.

${ }^{\mathrm{b}}$ Solution phase.

${ }^{\mathrm{c}}$ Vapor phase.

less, the comparison with the few well-assigned frequencies obtained from solution and vapor-phase experiments, ${ }^{35,36}$ given in Tables II and III, provides insight into the capabilities and precision of our approach. The assignment of Girlando and Pecile ${ }^{34}$ has been chosen for comparison, because it is complete and has been used extensively in the literature on CA crystals and charge-transfer compounds including CA. Their assignment of $b_{3 g} \nu_{12}$ and $\nu_{13}, b_{1 u} \nu_{20}$ and $\nu_{21}$, and $b_{3 u} \nu_{28}$ eigenfrequencies is confirmed by our results.

For the high-frequency modes our calculations show good agreement with experiment on CA crystals (with typically a $3 \%$ deviation). For the low-frequency modes around 200 $\mathrm{cm}^{-1}$ and less, the deviation is generally larger (more than $6 \%$ ). Nevertheless, this was foreseeable for two reasons. The first is numerical: owing to the finite simulation time $(\sim 1$ ps), only a few periods of the low-frequency modes are reproduced, so that these frequencies are estimated with a larger error than the high ones. The second argument is physical: in a crystal, low-frequency intramolecular modes are more strongly coupled to lattice vibration modes than high-frequency modes are, causing the deviation from our calculations for an isolated molecule. Finally, the eigenvector plots of all in-plane and out-of-plane modes in Figs. 5 and 6 provide a clear picture of the fundamental vibrations.

\section{INFLUENCE OF THE IONICITY}

Chloranil is a strong electron acceptor, and many experimental investigations have been performed on its radical salts. Vibrational and structural analysis of this molecule in known oxidation states can help understand the spectral and structural changes that occur when CA forms CT complexes or radical salts. In this part of the work, we have undertaken a thorough analysis of static and dynamical properties of two oxidation states $\left(+0.5 e^{-}\right.$and $\left.+1 e^{-}\right)$in comparison with the results for the neutral CA given in Sec. II.

\section{A. Structural changes}

For both oxidation states, the optimized geometry (obtained by a simulation at $0 \mathrm{~K}$ ) is planar and belongs to the $D_{2 h}$ molecular point group. The calculated bond lengths and angles are listed in Table IV. All four bond lengths change essentially linearly with the charge. The $\mathrm{C}=\mathrm{O}$ bond length is most strongly affected. The modification of the $\mathrm{C}-\mathrm{Cl}$ one is also appreciable, while that of the $\mathrm{C}-\mathrm{C}$ ones is less important. Some of these variations can be understood when looking at the LUMO of CA [Fig. 3(b)], which becomes occupied as an electron is added: this orbital is a combination of $\pi$ antibonds between $\mathrm{C}=\mathrm{O}$ and $\mathrm{C}-\mathrm{Cl}$. This is the origin for the increase of the $\mathrm{C}=\mathrm{O}$ and $\mathrm{C}-\mathrm{Cl}$ bond lengths.

To our knowledge, the only structural data available for $\mathrm{CA}^{-}$are those of Konno and co-workers in crystalline $\mathrm{K}^{+} \mathrm{CA}^{-38} \cdot{ }^{38}$ As their experimental error is at least half of our bond-length variations between $\mathrm{CA}^{0}$ and $\mathrm{CA}^{-}$, we refrain from a comparison. Nevertheless, another interesting comparison can be made with the experimental data available for CA in the TTF-CA crystal. ${ }^{39}$ This CT complex undergoes a neutral-to-ionic phase transition when the temperature is lowered below $\simeq 80 \mathrm{~K}$, and the CA molecule is observed to

TABLE IV. Calculated bond lengths (in $\AA$ ) and angles (in degrees) for various oxidation states of the chloranil molecule.

\begin{tabular}{lccccccccc}
\hline \hline Charge & $\mathrm{C}_{1}-\mathrm{O}$ & $\mathrm{C}_{1}-\mathrm{C}_{2}$ & $\mathrm{C}_{2}-\mathrm{C}_{3}$ & $\mathrm{C}_{2}-\mathrm{Cl}$ & $\mathrm{C}_{2} \mathrm{C}_{1} \mathrm{C}_{6}$ & $\mathrm{C}_{2} \mathrm{C}_{1} \mathrm{O}$ & $\mathrm{C}_{1} \mathrm{C}_{2} \mathrm{C}_{3}$ & $\mathrm{C}_{1} \mathrm{C}_{2} \mathrm{Cl}$ & $\mathrm{ClC}_{2} \mathrm{C}_{3}$ \\
\hline$+0.00 e^{-}$ & 1.219 & 1.477 & 1.350 & 1.671 & 117.9 & 121.1 & 121.1 & 116.3 & 122.6 \\
$+0.25 e^{-}$ & 1.227 & 1.471 & 1.356 & 1.678 & 116.8 & 121.6 & 121.6 & 116.1 & 122.3 \\
$+0.50 e^{-}$ & 1.236 & 1.464 & 1.364 & 1.681 & 115.6 & 122.2 & 122.2 & 115.9 & 121.9 \\
$+0.75 e^{-}$ & 1.243 & 1.456 & 1.367 & 1.690 & 115.1 & 122.4 & 122.4 & 115.9 & 121.7 \\
$+1.00 e^{-}$ & 1.251 & 1.453 & 1.374 & 1.698 & 114.6 & 122.7 & 122.7 & 115.8 & 121.4 \\
\hline \hline
\end{tabular}


TABLE V. Mean values of the chloranil bond lengths (in $\AA$ ) in the TTF-CA crystal for three different temperatures, obtained in neutron-scattering measurements (Ref. 39).

\begin{tabular}{rcccc}
\hline \hline$T(\mathrm{~K})$ & $\mathrm{C}_{1}-\mathrm{O}$ & $\mathrm{C}_{1}-\mathrm{C}_{2}$ & $\mathrm{C}_{2}-\mathrm{C}_{3}$ & $\mathrm{C}_{2}-\mathrm{Cl}$ \\
\hline 300 & 1.215 & 1.489 & 1.352 & 1.698 \\
90 & 1.219 & 1.486 & 1.359 & 1.706 \\
40 & 1.232 & 1.477 & 1.363 & 1.713 \\
\hline \hline
\end{tabular}

have a lesser charge at high temperatures than at low ones. In Table $\mathrm{V}$ we report the mean values of these experimental bond lengths at each temperature.

First of all, when going from 300 to $40 \mathrm{~K}$, all four distances behave in the same way as our calculated values do when going from $\mathrm{CA}^{0}$ to $\mathrm{CA}^{-}$. Furthermore, from the total bond-length changes between 300 and $40 \mathrm{~K}$ and our results, one can estimate the corresponding variation of the molecular ionicity $\Delta \rho$. The four bond-length types lead to the same estimate of $\Delta \rho=0.5 \pm 0.1$, which is in good agreement with previous estimates. ${ }^{9-11,39}$

\section{B. Vibrational changes}

For each oxidation state of CA $\left(+0.5 e^{-}\right.$and $\left.+1 e^{-}\right)$we have performed an MD simulation at $30 \mathrm{~K}$ for $1.5 \mathrm{ps}$. The symmetry of the vibrational modes was again treated in the context of the $D_{2 h}$ molecular point group, and a correlative assignment was made by comparison with the eigenvectors of the neutral molecule. The resulting frequencies are given in Tables VI and VII in comparison with experiments on KCA crystalline powders, in which a charge transfer of one electron from $\mathrm{K}$ to $\mathrm{CA}$ is generally assumed.

As found experimentally, there is no unique direction of shift for all fundamentals, and its magnitude varies from 0 to $200 \mathrm{~cm}^{-1}$. Furthermore, the mode mixing upon ionization ${ }^{40}$ of the upper two totally symmetric modes has also been observed: for the neutral molecule the upper frequency mode has a $\mathrm{C}-\mathrm{O}$ and the second mode a $\mathrm{C}-\mathrm{C}$ stretching character, whereas for the radical anion these characters are found to be interchanged. The vibrations most strongly affected by the change of ionicity are of C-O type $\left(a_{g} \nu_{1}-\nu_{2}, a_{g} \nu_{4}\right.$, and $\left.b_{1 u} \nu_{18}\right)$. Some of the C-C modes are also appreciably perturbed.

The calculation for $\mathrm{CA}+0.5 e^{-}$is interesting for two reasons. First, it is an additional tool to check the performance of this method. Secondly, at least three points are necessary to verify that the frequencies of interest vary linearly with ionicity qualitatively. The calculated frequencies in Tables VI and VII show that for those eigenmodes that present a significant shift, the assumption of linear behavior can readily be used, for example, to obtain a rough estimate of the molecular ionicity.

Nevertheless, one has to be careful when drawing a parallel between frequency shift and ionicity, as possible vibronic effects may affect the vibrational behavior considerably. These electron-intramolecular-vibration interactions cause an additional shift of the frequencies for modes that

TABLE VI. Calculated in-plane vibrational frequencies $\left(\right.$ in $\mathrm{cm}^{-1}$ ) for various oxidation states of the chloranil molecule compared to experimental results of chloranil potassium salt crystalline powders (Ref. 40) $\Delta$ represents deviation of the calculated values from the experimental results in Ref. 40. The last two columns give the calculated and experimental frequency shift (Refs. 34 and 40 ) between the neutral molecule and the radical anion, respectively.

\begin{tabular}{|c|c|c|c|c|c|c|c|c|}
\hline $\begin{array}{l}\text { Symm. } \\
\text { species }\end{array}$ & & Neutral & $\begin{array}{c}\text { CP-PAW } \\
+0.5 e^{-}\end{array}$ & $+1 e^{-}$ & $\begin{array}{l}\text { Ref. } 40 \\
+1 e^{-}\end{array}$ & $\Delta \%$ & $\begin{array}{c}\text { CP-PAW } \\
\text { shift }\end{array}$ & $\begin{array}{r}\text { Expt. } \\
\text { shift }\end{array}$ \\
\hline \multirow[t]{6}{*}{$a_{g}$} & $\nu_{1}$ & 1713 & 1629 & 1532 & 1518 & 1 & -181 & -175 \\
\hline & $\nu_{2}$ & 1588 & 1560 & 1535 & 1594 & 4 & -53 & -13 \\
\hline & $\nu_{3}$ & 1032 & 1039 & 1039 & 1028 & 1 & +7 & +21 \\
\hline & $\nu_{4}$ & 478 & 524 & 523 & 517 & 1 & +45 & +21 \\
\hline & $\nu_{5}$ & 328 & 339 & 336 & 338 & 1 & +8 & +8 \\
\hline & $\nu_{6}$ & 189 & 189 & 190 & & & +1 & \\
\hline \multirow{5}{*}{$b_{3 g}$} & $\nu_{11}$ & 1243 & 1278 & 1320 & 1368 & 4 & +77 & +121 \\
\hline & $\nu_{12}$ & 901 & 887 & 871 & 866 & 1 & -30 & +14 \\
\hline & $\nu_{13}$ & 747 & 744 & 738 & 760 & 3 & -11 & \\
\hline & $\nu_{14}$ & 336 & 332 & 332 & 318 & 5 & -4 & +11 \\
\hline & $\nu_{15}$ & 239 & 262 & 270 & 287 & 7 & +31 & +20 \\
\hline \multirow[t]{5}{*}{$b_{1 u}$} & $\nu_{18}$ & 1713 & 1617 & 1537 & 1525 & 1 & -176 & -160 \\
\hline & $\nu_{19}$ & 1155 & 1152 & 1157 & 1149 & 1 & -2 & +39 \\
\hline & $\nu_{20}$ & 951 & 953 & 950 & 918 & 4 & -1 & +10 \\
\hline & $\nu_{21}$ & 490 & 486 & 476 & 468 & 2 & -14 & -5 \\
\hline & $\nu_{22}$ & 210 & 194 & 196 & 200 & 2 & -14 & -14 \\
\hline \multirow[t]{5}{*}{$b_{2 u}$} & $\nu_{23}$ & 1531 & 1483 & 1439 & 1545 & 8 & -92 & -27 \\
\hline & $\nu_{24}$ & 1245 & 1174 & 1133 & $1175^{\mathrm{a}}$ & 4 & -112 & -85 \\
\hline & $\nu_{25}$ & 787 & 761 & 747 & 722 & 4 & -30 & -33 \\
\hline & $\nu_{26}$ & 391 & 365 & 357 & $374,369^{\mathrm{a}}$ & 5 & -34 & -2 \\
\hline & $\nu_{27}$ & 195 & 193 & 199 & 212 & 7 & +4 & +6 \\
\hline
\end{tabular}

${ }^{\mathrm{a}}$ Ref. 41 (KCA). 
TABLE VII. Calculated out-of-plane vibrational frequencies $\left(\mathrm{in}^{-1}\right.$ ) for various oxidation states of the chloranil molecule compared to experimental results of chloranil potassium salts crystalline powders. $\Delta$ represents the deviation of the calculated values from the experimental results in Ref. 40. The last two columns give the calculated and experimental frequency shift (Refs. 34 and 40) between the neutral molecule and the radical anion, respectively.

\begin{tabular}{|c|c|c|c|c|c|c|c|c|}
\hline $\begin{array}{l}\text { Symm. } \\
\text { species }\end{array}$ & & Neutral & $\begin{array}{c}\text { CP-PAW } \\
+0.5 e^{-}\end{array}$ & $+1 e^{-}$ & $\begin{array}{l}\text { Ref. } 40 \\
+1 e^{-}\end{array}$ & $\Delta \%$ & $\begin{array}{c}\text { CP-PAW } \\
\text { shift }\end{array}$ & $\begin{array}{l}\text { Expt. } \\
\text { shift }\end{array}$ \\
\hline$b_{1 g}$ & $\nu_{7}$ & 283 & 296 & 298 & & & +15 & \\
\hline \multirow[t]{3}{*}{$b_{2 g}$} & $\nu_{8}$ & 811 & 794 & 772 & & & -39 & \\
\hline & $\nu_{9}$ & 440 & 411 & 399 & & & -41 & \\
\hline & $\nu_{10}$ & 84 & 106 & 113 & & & +29 & \\
\hline \multirow[t]{2}{*}{$a_{u}$} & $\nu_{16}$ & 566 & 554 & 564 & & & -2 & \\
\hline & $\nu_{17}$ & 66 & 67 & 70 & & & +4 & \\
\hline \multirow[t]{3}{*}{$b_{3 u}$} & $\nu_{28}$ & 736 & 727 & 713 & 695 & 3 & -23 & -19 \\
\hline & $\nu_{29}$ & 169 & 173 & 174 & 220 & 27 & +5 & +34 \\
\hline & $\nu_{30}$ & 65 & 73 & 86 & $88^{\mathrm{a}}$ & 3 & +21 & \\
\hline
\end{tabular}

${ }^{\mathrm{a}}$ Ref. 41 (KCA).

can couple by symmetry with electrons. ${ }^{42}$ For example, in segregated CT complexes these interactions manifest themselves in the infrared spectra, ${ }^{43}$ whereas in mixed-stack TTF-CA crystals only the totally symmetric Raman-active $a_{g}$ modes are affected. ${ }^{9}$ Thus, in this latter crystal the $b_{1 u} \nu_{18}$ $\mathrm{C}-\mathrm{O}$ stretching mode of $\mathrm{CA}$ is not modified by vibronic effects, and its frequency shift can be used to estimate the jump in ionicity. From the data reported in Ref. 9, we again obtain a strong variation of the ionicity between the highand low-temperature phases of TTF-CA, namely $\Delta \rho \simeq 0.4$, which is in agreement with other estimates given in the literature.

\section{Coulomb repulsion and electron affinity}

Starting with our first-principles calculations, we now determine the relevant model parameters for electron-transfer processes towards a CA molecule in a CT complex. The LUMO of CA is the relevant orbital involved in such processes. The results of our density functional calculations in various charge and spin states are given in Table VIII.

In order to deduce the Coulomb repulsion and the spinsplitting parameters, we combine our calculations with the simple model energy function proposed by Carloni and co-workers. ${ }^{44}$ In this model the energy of an isolated species is given by

$$
\begin{aligned}
E\left[\Delta n_{\uparrow}, \Delta n_{\downarrow}\right]= & \bar{E}+\bar{\epsilon}\left(\Delta n_{\uparrow}+\Delta n_{\downarrow}\right)+\frac{1}{2} U\left(\Delta n_{\uparrow}+\Delta n_{\downarrow}\right)^{2} \\
& -\frac{1}{2} J\left(\Delta n_{\uparrow}-\Delta n_{\downarrow}\right)^{2}
\end{aligned}
$$

where $\bar{E}$ is the total energy of the reference state, $\bar{\epsilon}$ the energy of the relevant orbital in the reference state, $U$ the Coulomb repulsion of an electron in that orbital, $J$ the spinsplitting parameter or exchange coupling, and $\left(\Delta n_{\uparrow}, \Delta n_{\downarrow}\right)$ its occupation relative to the reference state.

First, we start with a set of non-spin-polarized calculations (in which the occupations satisfy $n_{\uparrow}=n_{\downarrow}$ ). The corresponding estimates of $U$ for each occupation when taking the empty LUMO state as reference are given in Table VIII. We obtain a Coulomb repulsion of the order of $4.8 \mathrm{eV}$ for the LUMO state. For the completely neutral or charged molecule, we also performed calculations including gradient corrections. Both these calculations with $\mathrm{GC} 1$ and $\mathrm{GC} 2$ gave a similar result: the corresponding estimate for $U$ was 4.7 eV.

From the expansion of $E\left[\Delta n_{\uparrow}, \Delta n_{\downarrow}\right]$ one can estimate the spin-splitting parameter $J$ independently from the estimate of the Coulomb repulsion $U$. As reference state we take the equal occupations of half an electron for each spin state in the LUMO and compare it to spin-polarized calculations for $\mathrm{CA}^{-}$with different occupations in the two spin states. The two estimates given in the last lines of Table VIII yield a value of $0.16 \mathrm{eV}$ for $J$. The same value can also be deduced from the difference between the spin-up and spin-down energy levels.

An estimate of the electron affinity of CA can be obtained either from the difference in total energy for the neutral molecule and the radical anion, or from the mean value of the LUMO energy when empty or occupied by one electron. Our non-spin-polarized LDA calculations yield 2.83 and $2.87 \mathrm{eV}$, respectively, which compares well with the experimental value of $2.76 \pm 0.2 \mathrm{eV}{ }^{45}$ Furthermore, the use of gradient

TABLE VIII. Calculated total energy and energy of the LUMO state for different total or spin-up and spin-down occupations of the LUMO state(s). Deduced values for the Coulomb repulsion $U$ and the spin-splitting parameter $J$ as obtained by using Eq. (4). All energies are given in $\mathrm{eV}$.

\begin{tabular}{lcccc}
\hline \hline Occupations $\left(n_{\uparrow}, n_{\downarrow}\right)$ & $\epsilon_{\mathrm{LUMO}}$ & $E_{\mathrm{tot}}$ & $U$ & $J$ \\
\hline$n_{\uparrow}=n_{\downarrow}=0.0$ & -5.213 & -3465.4620 & & \\
$n_{\uparrow}=n_{\downarrow}=0.125$ & -4.036 & -3466.6165 & 4.76 & \\
$n_{\uparrow}=n_{\downarrow}=0.25$ & -2.885 & -3467.4731 & 4.76 & \\
$n_{\uparrow}=n_{\downarrow}=0.375$ & -1.709 & -3468.0339 & 4.76 & \\
$n_{\uparrow}=n_{\downarrow}=0.5$ & -0.536 & -3468.2958 & 4.76 & \\
$n_{\uparrow}=0.25$ & -0.454 & -3468.3159 & & 0.16 \\
$n_{\downarrow}=0.75$ & -0.617 & & & \\
$n_{\uparrow}=0.0$ & -0.374 & -3468.3768 & & 0.16 \\
$n_{\downarrow}=1.0$ & -0.700 & & & \\
\hline \hline
\end{tabular}


corrections or spin-polarized calculations does not lead to a drastic change of this estimate: GC1 or spin polarization yields an increase of about $3-4 \%$, and GC2 results in a value that is $3 \%$ smaller than the estimate.

\section{CONCLUSION}

Using first-principles calculations we have thoroughly described the ground states and vibrations of chloranil molecule. By considering different oxidation states of this molecule, we were able to demonstrate that its bond lengths and vibrational frequencies change almost linearly with molecular ionicity. This provides the first theoretical confirmation of this linearity, which is a fundamental (but so far unverified) assumption for the experimental determination of molecular ionicity in CT compounds. An estimate for the Coulomb repulsion, the spin-splitting parameter, and the electron affinity have also been deduced. Whenever possible, comparison with experiments was made and an overall good agreement found.

\section{ACKNOWLEDGMENTS}

This work is part of the Joint Research Project (No. 82280512) between the two research partners of this article. Moreover, it has benefited from collaborations within, and has been partially funded by, the Human Capital Mobility Network on "Ab initio (from electronic structure) calculation of complex processes in materials" (Contract No. ERBCHRXCT930369). Part of the calculations have been supported by the "Institut du Développement et des Ressources Informatiques Scientifiques" of CNRS France.
${ }^{1}$ J. Ferraris, D. O. Cowan, V. Walatka, and J. Perlstein, J. Am. Chem. Soc. 95, 948 (1973).

${ }^{2}$ L. B. Coleman, M. J. Cohen, D. J. Sandman, F. G. Yamagishi, A. F. Garito, and A. J. Heeger, Solid State Commun. 12, 1125 (1973)

${ }^{3}$ R. Bozio and C. Pecile, in The Physics and Chemistry of Low Dimensional Solids, edited by L. Alcacer (Reidel, Dordrecht, 1980).

${ }^{4}$ J. B. Torrance, J. E. Vasquez, J. J. Mayerle, and V. Y. Lee, Phys. Rev. Lett. 46, 253 (1981).

${ }^{5}$ J. Yarwood, in Spectroscopy and Structure of Molecular Complexes, edited by J. Yarwood (Plenum, London, 1973), p. 184.

${ }^{6}$ Z. G. Soos, H. J. Keller, W. Moroni, and D. Nöthe, Ann. N.Y. Acad. Sci. 313, 442 (1978).

${ }^{7}$ Z. G. Soos and S. R. Bodenson, in Extended Linear Chain Compounds, edited by J. S. Miller (Plenum, New York, 1982), Vol. 3, p. 193 .

${ }^{8}$ T. C. Umland, S. Allie, T. Kuhlmann, and P. Coppens, J. Phys. Chem. 92, 6456 (1988).

${ }^{9}$ A. Girlando, F. Marzola, and C. Pecile, J. Chem. Phys. 79, 1075 (1983)

${ }^{10}$ C. S. Jacobsen and J. B. Torrance, J. Chem. Phys. 78, 112 (1983).

${ }^{11}$ A. Girlando, A. Painelli, and C. Pecile, Mol. Cryst. Liq. Cryst. 120, 17 (1985)

${ }^{12}$ Y. Tokura, H. Okamoto, T. Koda, T. Mitani, and G. Saito, Solid State Commun. 57, 607 (1986).

${ }^{13}$ TTF: tetrathiafulvalene $\left(\mathrm{C}_{6} \mathrm{~S}_{4} \mathrm{H}_{4}\right)$; DMTTF: 2,6-dimethyltetrathiafulvalene $\left(\mathrm{C}_{8} \mathrm{~S}_{4} \mathrm{H}_{8}\right)$; TMPD: tetramethylparaphenylenediamine $\left(\mathrm{C}_{10} \mathrm{~N}_{2} \mathrm{H}_{16}\right)$.

${ }^{14}$ P. E. Blöchl, Phys. Rev. B 50, 17953 (1994).

${ }^{15}$ R. Car and M. Parrinello, Phys. Rev. Lett. 55, 2471 (1985).

${ }^{16}$ R. Car and M. Parrinello, in Simple Molecular Systems at Very High Density, edited by A. Polian, P. Loubeyre, and N. Boccara (Plenum, New York, 1989), p. 455.

${ }^{17}$ P. Margl, K. Schwarz, and P. E. Blöchl, J. Chem. Phys. 100, 8194 (1994).

${ }^{18}$ O. K. Andersen, Phys. Rev. B 12, 3060 (1975).

${ }^{19}$ D. R. Hamann, M. Schlüter, and C. Chiang, Phys. Rev. Lett. 43, 1494 (1979).

${ }^{20}$ P. Margl, Ph.D. thesis, TU Vienna, Austria, 1994.
${ }^{21}$ J. Kohanoff, Comput. Mater. Sci. 2, 221 (1994); J. Kohanoff, W. Andreoni, and M. Parrinello, Phys. Rev. B 46, 4371 (1992).

${ }^{22}$ J. P. Perdew and A. Zunger, Phys. Rev. B 23, 5048 (1981).

${ }^{23}$ M. Ceperley and B. L. Adler, Phys. Rev. Lett. 45, 566 (1980).

${ }^{24}$ A. D. Becke, J. Chem. Phys. 96, 2155 (1992).

${ }^{25}$ J. P. Perdew, Phys. Rev. B 33, 8822 (1986).

${ }^{26}$ P. E. Blöchl, J. Chem. Phys. 103, 7422 (1995).

${ }^{27}$ S. Nosé, J. Chem. Phys. 81, 511 (1984).

${ }^{28}$ S. Nosé, Mol. Phys. 52, 255 (1984).

${ }^{29}$ P. E. Blöchl and M. Parrinello, Phys. Rev. B 45, 9413 (1992).

${ }^{30}$ L. Verlet, Phys. Rev. 159, 98 (1967).

${ }^{31}$ K. J. Van Weperen and G. J. Visser, Acta Crystallogr. B 28, 338 (1972).

${ }^{32}$ S. S. S. Chu, G. A. Jeffrey, and T. Sakukai, Acta Crystallogr. 15, 661 (1962)

${ }^{33}$ H. Yamada, M. Saheki, S. Fukushima, and T. Nagaosa, Spectrochim. Acta 30A, 295 (1973).

${ }^{34}$ A. Girlando and C. Pecile, J. Chem. Soc. Faraday Trans. 69, 1291 (1973).

${ }^{35}$ F. E. Prichard, Spectrochim. Acta 20, 127 (1964).

${ }^{36}$ N. E. Sharpless and D. A. Gregory, Appl. Spectrosc. 17, 47 (1963).

${ }^{37}$ H. Yamada and M. Kawamori, Spectrochim. Acta 27A, 2425 (1971).

${ }^{38}$ M. Konno, H. Kobayashi, F. Marumo, and Y. Saito, Bull. Chem. Soc. Jpn. 46, 1987 (1973).

${ }^{39}$ M. Le Cointe, M. H. Lemée-Cailleau, H. Cailleau, B. Toudic, L. Toupet, G. Heger, F. Moussa, P. Schweiss, K. H. Kraft, and N. Karl, Phys. Rev. B 51, 3374 (1995).

${ }^{40}$ A. Girlando, I. Zamon, R. Bozio, and C. Pecile, J. Chem. Phys. 68, 22 (1978).

${ }^{41}$ A. Girlando, L. Morelli, and C. Pecile, Chem. Phys. Lett. 22, 553 (1973).

${ }^{42}$ C. Pecile, A. Painelli, and A. Girlando, Mol. Cryst. Liq. Cryst. 171, 69 (1989).

${ }^{43}$ A. Girlando, R. Bozio, and C. Pecile, Phys. Rev. B 26, 2306 (1982).

${ }^{44}$ P. Carloni, P. E. Blöchl, and M. Parrinello, J. Phys. Chem. 99, 1338 (1995).

${ }^{45}$ C. D. Cooper, W. F. Frey, and R. N. Compton, J. Chem. Phys. 69, 2367 (1978). 